\title{
Les fonctionnaires et la fabrique des politiques publiques : simples figurants ou véritables acteurs?
}

\section{Dominique Boily et Jean-François Savard}

École nationale d'administration publique, Pavillon Alexandre-Taché, aile D, 283, boulevard Alexandre-Taché, Case postale 1250, succursale Hull, J8R3N5 Gatineau, Canada

\section{Résumé}

Une conception wébérienne, idéalisée et monolithique, du fonctionnaire, neutre et apolitique pourrait nous faire négliger d'étudier son rôle dans le continuum des politiques publiques. En effet, si le rôle du fonctionnaire se limite à celui du robot, son action n'aurait aucun effet sensible sur l'action de l'État. La réalité est pourtant beaucoup plus complexe et souvent en porte-à-faux avec cette image d'Épinal. Le présent article se penche sur ces acteurs-fonctionnaires et réfléchis sur l'impact significatif qu'ils peuvent avoir sur la mise en œuvre des politiques publiques. Conditionnés par des institutions internes qui ne s'inscrivent pas toujours dans un mouvement linéaire qui serait en symbiose avec la finalité de l'action publique globale, les propres déterminants culturels des fonctionnaires déterminent une bonne partie de leurs actions. L'effet de leur rencontre, à l'intersection de mondes trop souvent parallèles, constitue un facteur non négligeable de la fabrique des politiques publiques.

(c) 2017 IDMP/Lavoisier SAS. Tous droits réservés

Mots clés : acteurs, règles, néo-institutionnalisme, mise en œuvre, politiques publiques.

\section{Abstract}

\section{Public Servants and Public Policy Manufacturing: Simple Extras or Eeal Actors ? A}

Weberian, idealized and monolithic conception of the neutral and apolitical civil servant could lead us to neglect studying its role in the continuum of public policy. Indeed, if the public servant's role is limited a robot-like, his action would have no appreciable effects on the State's action. The reality is, however, much more complex and often at odds with this ideal image. This article looks at these public servants-actors and reflects on the significant impact they can have on the implementation of public policies. Conditioned by internal institutions that do not always fit in a linear movement that would be in symbiosis with the purpose of global 
public action, public servants' cultural characteristics determine a good part of their actions. The effect of their encounter, at the intersection of different worlds that are too often parallel, is a contributing factor in public policy manufacturing. @ 2017 IDMP/Lavoisier SAS. Tous droits réservés

Keywords: actors, rules, neo-institutionalism, implementation, public policy.

\section{Introduction}

La conception idéalisée du fonctionnaire, neutre, apolitique, mettant en œuvre de manière quasi-robotique les ordres reçus doit beaucoup aux principes de Weber (Labolo, 2013). Si on accepte ce portrait monolithique du fonctionnaire à titre d'acteur, il n'y aurait que peu d'intérêt à étudier son rôle dans le continuum des politiques publiques. En effet, dans cette image idéalisée, le fonctionnaire n'ayant aucun apport, volontaire ou involontaire, sur le produit, l'étude de son rôle ne pourrait dépasser celle d'un simple conducteur, neutre et impassible, sans effet sensible sur l'action de l'État. La réalité est en effet beaucoup plus complexe et l'administration publique, appuyée par la sociologie et la psychologie, de même que par d'autres disciplines, a remis en cause cette image d'Épinal (Perry er Wise, 1990).

Le présent article se penche sur ces acteurs-fonctionnaires et sur l'impact qu'ils peuvent avoir sur la mise en œuvre des politiques publiques. En comprenant mieux ces jeux complexes, nous pourrons décrypter davantage ce qui se passe dans la fabrique des politiques publiques.

\section{Les acteurs dans la fabrique des politiques publiques}

Depuis plus de vingt ans, la question des acteurs dans les processus de politiques publiques - de la conception à la mise en œuvre - a été un thème récurrent dans tous les ouvrages portant sur les politiques publiques. Certains cherchent d'abord à proposer des typologies d'acteurs. C'est le cas, par exemple de Howlett et Ramesh (1995) qui reconnaissent cinq groupes d'acteurs à savoir les élus - qui comprennent les membres de l'exécutif et les membres du législatif, les fonctionnaires, les groupes d'intérêts, les organisations de recherche et les médias. Cette typologie de Howlett et Ramesh se fonde sur les fonctions que des groupes d'acteurs occupent au sein des processus de politique publique. Cette typologie est intéressante, mais elle demeure limitée, puisqu'elle ne permet pas de déterminer comment un acteur ou un groupe qui ne se fédère pas à l'une de ces catégories peut s'inscrire dans la fabrique des politiques.

Lemieux (2002), propose une approche permettant de dépasser cette limite qui se base sur deux critères : la perspective de l'acteur et la possession des ressources. L'auteur distingue ainsi les acteurs qui se trouvent à l'intérieur de l'appareil gouvernemental, de ceux qui se trouvent à l'extérieur et peuvent posséder des ressources statutaires non spécialisées ou des ressources informationnelles spécialisées. Ces quatre critères mènent Lemieux à reconnaître quatre grandes catégories d'acteurs : les responsables (ex. les élus), les agents (ex. les fonctionnaires), les particuliers (ex. groupes de citoyens) et les intéressés (ex. les syndicats, les groupes d'intérêts, les organisations de recherche). Birkland (2005) analyse le rôle des acteurs selon deux perspectives, à savoir les acteurs officiels (élus, fonctionnaires) 
et les acteurs non officiels (groupes d'intérêts, citoyens), alors que Bernier et Lachapelle (1998) divisent les acteurs selon deux groupes que sont les acteurs formels (politiciens élus, ministres, premiers ministres, fonctionnaires) et les acteurs politiques (groupes d'intérêts, citoyens, médias). Notons enfin que Knoepfel et coll. (2015) conçoivent la division des acteurs selon leur rôle dans un processus de politiques publiques, cette division prenant la forme d'un triangle qui unit les autorités politico-administratives (élus, gouvernements et fonctionnaires), les bénéficiaires finaux (acteurs affectés par les enjeux publics) et les groupes cibles (groupes d'acteurs visés par l'intervention d'une politique publique au profit des bénéficiaires finaux).

Ensuite, certains auteurs cherchent davantage à comprendre comment agissent les acteurs dans un processus de politiques publiques que de les catégoriser. Émergent ainsi les notions de ressources (Muller et Surel, 1998), qui déterminent les actions possibles des groupes d'acteurs et de répertoire d'actions qui visent à déterminer les préférences de l'agir des acteurs (Pal, 2006). Toutefois, plusieurs travaux ont fait ressortir la difficulté d'isoler les impacts que des acteurs donnés ont sur un processus de politiques publiques et ont démontré le caractère de plus en plus ficelé de l'action dans les processus des politiques publiques. C'est ainsi que certains auteurs se sont penchés sur l'étude des coalitions d'acteurs (Sabatier, 1999) et de réseaux (Lemieux, 2002; Grossman, 2014, Thatcher 2004). L'approche des coalitions suggère qu'à l'intérieur d'un sous-système de politiques publiques, les acteurs aient tendance à former des coalitions, que Sabatier (1999) caractérise de « plaidante », qui sont formées d'acteurs provenant de différentes organisations, partageant certaines croyances et agissant de façon concertée. Comme certaines coalitions pourraient se trouver dans des situations de conflits, des médiateurs s'assurent d'arbitrer ces conflits pour ne pas nuire à l'action concertée (Lemieux, 2002).

Lemieux (2002) ne nie pas le bien-fondé de l'approche des coalitions, mais il suggère de l'utiliser avec celle des alliances. Les alliances sont des regroupements plus larges, mais qui englobent les coalitions. Les alliances constituent des ensembles plus ou moins conjoncturels et plus ou moins négociées d'acteurs individuels ou collectifs qui ont à la fois des rapports de coopération et de conflits. Ces acteurs cherchent à obtenir des avantages plus grands que s'ils ne faisaient pas partie de l'alliance.

L'approche des réseaux « [...] propose un schéma d'interprétation des relations Étatsociété qui met l'accent sur le caractère horizontal et non hiérarchique de ces relations, le caractère relativement informel des échanges entre les acteurs du réseau, l'absence de fermeture qui autorise la multiplication des échanges périphériques et la combinaison de ressources techniques [...] et de ressources politiques [...]» (Muller et Surel, 1998 : p. 91). L'étude des réseaux s'intéresse aux relations entre les acteurs et à l'existence d'interdépendances entre ces derniers (Grossman, 2014). Un accent plus particulier est aussi porté vers les relations entre les groupes d'intérêts et de l'État (Thatcher, 2004). L'approche des réseaux constate que le processus de définition des politiques publiques est fragmenté et que les groupes d'intérêts participent activement à la prise de décision. En mettant ainsi l'accent sur les relations entre l'État et la société civile, l'approche des réseaux introduit une autre notion, celle de gouvernance.

La notion de gouvernance se présente comme un « processus de coordination d'acteurs, de groupes sociaux, d'institutions, en vue d'atteindre des objectifs définis et discutés collectivement » (Le Galès, 2014 : p. 301). La gouvernance est un processus négocié qui 
apparaît comme un mécanisme permettant aux acteurs publics et privés d'arriver à des décisions qui sont mutuellement satisfaisantes et contraignantes en vue de résoudre des conflits par la coopération (Le Galès, 2014). La négociation est donc l'engagement des acteurs privés et l'ouverture des acteurs publics à une participation mutuelle au sein d'un même processus de décision pour définir les politiques et les réglementations qui régissent un secteur particulier de la société.

\section{Du néo-institutionnalisme au contournement des règles : comment agit l'acteur- fonctionnaire dans la fabrique des politiques publiques}

Ce bref tour d'horizon des écrits universitaires montre bien que si l'existence des fonctionnaires comme acteurs dans les processus des politiques publiques est généralement reconnue, les travaux se sont presque exclusivement concentrés sur l'interaction entre les acteurs de la société civile et les élus ou les administrateurs publics. Très peu de travaux ont porté sur l'impact des interactions entre fonctionnaires dans la fabrique des politiques publiques. Cet article vise à mieux comprendre cet impact en se penchant sur la question suivante : comment les interactions entre fonctionnaires au sein d'une administration publique influencent-elles la fabrique des politiques publiques?

Pour ce faire, nous proposons de jeter un regard sur l'impact qu'ont les relations entre fonctionnaires dans la fabrique des politiques publiques sous l'angle néo-institutionnel. Fondé par March et Olson (1989), le néo-institutionnalisme prend racine dans une remise en question du rôle des institutions qui a été adoptée dans l'analyse des politiques publiques. De façon générale, le néo-institutionnalisme soutient que les acteurs et les institutions coexistent et s'influencent mutuellement (Lecours, 2002). Plus précisément, les institutions conditionnent l'action politique, définissant les cadres où se déploient les comportements des acteurs. Pour Muller et Surel (1998), le néo-institutionnalisme s'articule autour de deux éléments fondamentaux. D'abord, les institutions constituent des facteurs d'ordre à travers une multitude de règles qui conditionnent l'action politique (ex. règle de l'autorité, règle d'évaluation, règle de l'accès et de l'utilisation de l'information). Ce qui ne signifie pas que l'action politique soit déterminée par les institutions ou que les acteurs ne bénéficient pas de liberté dans le processus de politiques publiques. Bien au contraire « [...] parce que les règles sont multiples (et souvent contradictoire) [elles offrent] ainsi aux acteurs des nombreuses occasions de choix » (Muller et Surel, 1998). Ensuite, le néo-institutionnalisme conçoit la politique comme une interprétation du monde. Ainsi, les institutions ne sont pas que règles assurant l'ordre dans le système politique, elles relèvent également de la culture et de symboles permettant ainsi aux acteurs de donner un sens à leurs actions (Muller et Surel, 1998).

Le néo-institutionnalisme ne constitue toutefois pas une approche homogène, alors que l'on reconnaît généralement trois types de néo-institutionnalisme (Knoepfel et coll., 2015; Lecours, 2002) : l'institutionnalisme historique, l'institutionnalisme du choix rationnel et l'institutionnalisme sociologique. L'institutionnalisme historique repose sur le concept de path dependency selon lequel les institutions sont influencées par des facteurs contextuels et exogènes aux acteurs qui leur ont donné vie et produisent des dynamiques qui leur sont propres et surtout imprévues par les acteurs (Lecours, 2002). Réjean Pelletier précise que 
l'institutionnalisme historique montre comment les acteurs sont forcés de s'adapter aux institutions (Pelletier, 2005).

L'institutionnalisme du choix rationnel suppose qu'en tenant compte des éléments institutionnels dans l'analyse des politiques publiques « [...] on peut mieux rendre compte des choix individuels et du produit collectif tout en restant fidèle aux idées de rationalité et de recherche du gain personnel » (Lecours, 2002 : p. 9). Autrement dit, l'institutionnalisme du choix rationnel est moins déterministe que l'institutionnalisme historique reconnaissant que l'individu a toujours la liberté de choisir ses actions, mais dans les limites imposées par le contexte institutionnel (Pelletier, 2005).

L'institutionnalisme sociologique intègre dans son analyse des variables culturelles pour mieux comprendre les conditions de formation et le fonctionnement des organisations. Au-delà des règles, ce type d'analyse conçoit les éléments culturels comme des institutions qui définissent le cadre dans lequel se déploient les stratégies d'actions des acteurs impliqués dans les processus de politiques publiques. À l'image de Lecours (2002), nous dirons que l'institutionnalisme sociologique suppose un lien symbiotique entre les institutions et la société. Ainsi, l'institutionnalisme sociologique conçoit les institutions comme des constructions sociales, lesquelles sont créées et modifiées par les relations sociales et la perception qu'ont les acteurs du bien commun. Pour l'institutionnalisme sociologique, c'est dans la relation structure-agent que les institutions exercent leur influence sur l'action en définissant les comportements socialement acceptables (Lecours, 2002). Comme porteuses de codes culturels et cognitifs, les institutions génèrent, reproduisent et définissent les frontières du politique en déterminant pour les acteurs ce qui constitue l'action légitime.

C'est dans cette dernière approche que s'inscrit notre analyse de l'impact des relations entre fonctionnaires dans la fabrique des politiques publiques. Nous croyons en effet qu'au sein d'une administration publique, les fonctionnaires constituent des acteurs dont les comportements sont conditionnés à la fois par des règles, par l'esprit des règles et par un ensemble de codes culturels divers qui peuvent souvent être conflictuels en fonction du rôle que les acteurs occupent dans une administration publique. À cet égard, Katzenstein (1996) soutient que le conditionnement de l'acteur dans sa relation avec les politiques publiques vient d'un ensemble de normes collectives qui vont informer son comportement. Ces normes peuvent venir, entre autres, de la culture de l'organisation. On parlera dès lors de normes institutionnelles façonnant le comportement de l'acteur (Katzenstein, 1996).

Au sein d'une administration publique, les fonctionnaires appartiennent à des mondes culturels différents. Leurs comportements seront conditionnés par des règles et des codes culturels incompatibles qu'ils considèrent toutefois comme légitimes. C'est de cet état de fait que naissent alors des conflits qui auront des impacts dans la fabrique des politiques publiques. Pour y remédier, les uns auront tendance à imposer des règles et les autres à chercher à les contourner. C'est pourquoi, bien que l'acteur soit conditionné, nul comportement n'est parfaitement inéluctable, et il est toujours une circonstance où il pourra être influencé d'une façon ou d'une autre à agir concrètement à l'encontre de son conditionnement. Potocki Malicet (1997), dans une étude sur l'application des règles dans le milieu universitaire, conclut :

«Toute organisation est un lieu d'équilibre entre les buts qui lui sont propres et les buts de ceux qui la constituent. Quand une règle ne permet pas d'effectuer les activités prescrites de façon adéquate, la pression qui naîtra de cette situation dysfonctionnelle n'aboutira 
pas à l'abandon de cette règle, mais elle fera l'objet d'aménagements de la part des acteurs. » (Potocki Malicet, 1997, p. 68)

Cette interprétation se base sur la théorie de Bourdieu (1980), qui a établi qu'une règle officielle ne peut avoir d'effet sur les individus que lorsque l'intérêt à la respecter l'emporte sur l'intérêt à la contredire. Dès qu'il semble y avoir plus d'avantages à désobéir à une règle qu'à y obéir, l'adhésion à la règle se modifie et, comme l'a si bien dit Potocki Malicet (1997), sans pouvoir changer la règle, on y cherche désormais des aménagements.

Or, l'acteur n'agit pas seul. Il fait partie d'un groupe, et l'individu cherche constamment à « être en règle » avec le groupe (Bourdieu, 1980). Il faudra donc que le groupe lui-même voie un avantage plus grand à désobéir à la règle qu'à la respecter pour que cette recherche d'accommodements se réalise. Il s'agit donc d'une question de calcul de « coût/bénéfice » où l'acteur, comme membre d'un groupe, cherche à faire ce qui lui paraît le mieux, et le plus légitime, et même parfois contre la règle établie. Encore faut-il voir quel serait, dans le cas des règles institutionnelles, ce « mieux » qui forcerait l'acteur à agir ainsi.

Potocki Malicet (1997) croit pour sa part que les organisations disposent d'une certaine force interne qui les pousse à faire preuve de créativité en vue d'atteindre les objectifs de l'organisation. Ce serait cette énergie interne, qui permettrait aux acteurs de faire preuve de créativité et de contourner les règles établies dans le but de satisfaire aux raisons d'existence de l'organisation. Pour Bourdieu (1980), même si l'acte n'est pas forcément conscient, l'acteur finira toujours par intégrer ce que la scolastique a appelé une attention objective. Les acteurs cherchent, au fond, le bien de l'organisation.

Ce cadre théorique peut se synthétiser de la façon suivante. Aux prises à des règles qui lui sont imposées par un confrère, mais qui sont incompatibles avec un comportement qu'il juge légitime, l'acteur-fonctionnaire cherchera à contourner la règle soit en la réinterprétant pour en modifier le sens ou soit en l'ignorant afin d'atteindre le résultat visé. L'évolution de l'équilibre interne au sein des administrations publiques refléterait alors non seulement un changement stratégique au sein de l'organisation - soit la mise en place de jeux d'acteur nouveaux - , mais également un changement culturel au sein de l'institution.

\section{De la théorie au terrain : observation de l'impact sur les politiques publiques}

Deux études de cas doivent nous servir à illustrer l'évolution de la gouvernance interne de l'administration publique. Au sens premier, l'observation «... est un processus incluant l'attention volontaire et l'intelligence orientée par un objectif terminal ou organisateur et dirigé sur un objet pour en recueillir des informations. » (De Ketele, 1980, p. 27). Pour De Ketele et Roegiers (2009), l'observation demande la sélection d'un petit nombre d'informations pertinentes au sein de tous les possibles et requiert un cadre théorique de référence. De plus, le choix du terrain d'observation doit être fortement lié à la question de recherche (Arborio, 2007).

Chacun des cas est présenté avec un contexte et une description des relations entre les acteurs au moment A, alors qu'une crise de gouvernance est diagnostiquée et une description de la relation entre les acteurs au moment $\mathrm{B}$, après que de nouvelles relations de travail se soient établies.

Pour ce faire, nous nous sommes penchés sur le cas du ministère fédéral canadien de 
l'Environnement (que l'on appelle au pays Environnement Canada) ${ }^{2}$. Ce choix s'explique du fait qu'à la suite d'un ensemble de circonstances, le ministère a profondément modifié la façon de livrer ses services de ressources humaines passant d'une fonction tournée vers l'observation à la lettre des règles institutionnelles, à un mode de soutien stratégique aux politiques publiques. Le présent article ne nous permet pas d'entrer dans les détails des raisons qui expliquent ce changement. Cependant, ce double moment (avant et après le changement) nous permet de comparer deux types de relations entre ces acteurs et mieux comprendre comment ces relations (ou ces jeux d'acteurs) ont influencé la fabrique des politiques publiques. Les acteurs que nous avons choisi d'étudier sont composés de deux groupes : les gestionnaires et les conseillers en ressources humaines. Les gestionnaires sont ceux qui, en raison du mandat qui leur est confié, ont la responsabilité de mettre en œuvre des programmes liés à des politiques publiques. De l'autre côté, les conseillers en ressources humaines administrent des règles internes et conseillent les gestionnaires sur les décisions de ressources humaines en fonction de ces règles internes.

Afin d'illustrer les dynamiques observées entre les acteurs et leurs impacts sur les politiques publiques, deux cas ont été retenus pour leur clarté et leur simplicité. Clarté, puisqu'ils illustrent bien les conséquences concrètes de l'application des règles internes sur les politiques publiques et simplicité parce que le contexte peut être compris par un lecteur qui n'a pas un savoir technique avancé. L'observation, comme telle, a été faite à l'aide d'une prise de notes, au moment des faits, qui détaillait la nature du désaccord entre les acteurs, les raisons invoquées par chaque partie, et le détail de la résolution du désaccord.

\subsection{Premier cas : Langues officielles à Gagetown}

\section{Contexte}

Le gouvernement canadien doit s'assurer que les citoyens, l'industrie du transport, les forces de sécurité de même que ses forces armées disposent d'informations météo précises, adaptées à leurs besoins, et ce, en temps opportun. Ces informations, qu'elles soient sous forme de données brutes, de prévisions automatisées à court ou à long terme, d'analyses poussées ou d'images en temps réel, doivent être produites et communiquées, et leur accès doit être facilité. Pour ce faire, le Centre météorologique canadien d'Environnement Canada compte sur des actifs scientifiques et techniques ainsi que sur un personnel scientifique et administratif réparti dans des centres spécialisés partout au pays.

Les Forces armées canadiennes constituant l'un de ses principaux clients, le ministère a accepté de déménager un centre de météo militaire, basé à Toronto, vers une base d'hélicoptères militaires située près de Gagetown au Nouveau-Brunswick.

Ce déménagement fut compliqué en matière de disponibilité de la main-d'œuvre étant donné deux facteurs. Premièrement, l'essentiel de la force de travail d'un centre météo est composé de scientifiques, hautement spécialisés, qu'on ne retrouve qu'au sein du Ministère. Deuxièmement, Fredericton, la capitale provinciale, située à environ $60 \mathrm{~km}$, représente un centre relativement éloigné avec une population totale de seulement 56000 habitants.

\footnotetext{
2 Étant donné qu'un des auteurs du présent article travaillait au sein de la direction de ressources humaines, il a pu observer de près de très nombreux cas et noter lesdites observations pour une utilisation ultérieure de recherche.
} 


\section{Moment A}

Cette situation, déjà pleine de défis à l'égard des ressources humaines, fut exacerbée par l'application d'une règle qui veut que tous les employés des régions désignées bilingues, comme c'est le cas pour Gagetown, puissent travailler dans la langue officielle de leur choix. Or, d'une part, la nature du travail de ce centre, à savoir la production de prévisions météorologiques pour des pilotes, ne requiert que l'anglais. D'autre part, aucun employé francophone n'avait demandé de travailler dans cette langue. Il n'existait donc aucune justification in situ qui exigeait le bilinguisme de tous les gestionnaires.

Comme le centre de météo de Gagetown ne disposait pas d'un nombre suffisant de superviseurs bilingues pour répondre aux exigences de la règle, les conseillers en ressources humaines (acteur 2) conseillèrent d'envoyer la presque totalité des superviseurs en formation linguistique à temps plein au cours des prochaines années. À contrecœur, la gestion (acteur 1) obtempéra, non sans exprimer leurs frustrations. En effet, l'absence de plusieurs superviseurs créa une pression supplémentaire sur une force de travail déjà en nombre limité et compliqua les opérations pour un client qui n'avait aucunement besoin de services bilingues. Concrètement, cette pression sur la force de travail réduisait la flexibilité de la gestion dans l'assignation des quarts de travail d'un service qui doit fonctionner 24 heures sur 24, 365 jours par année. Cette situation mettait à risque la capacité opérationnelle du ministère de fournir le service à l'armée en tout temps. On s'exposait ainsi à des bris de service potentiel en opposition directe avec la politique publique. Aussi, lorsque les gestionnaires du service météo exprimaient leur frustration concernant cette situation, ils exprimaient les difficultés à mettre en œuvre la politique publique pertinente.

\section{Moment B}

Pour donner suite à la pression effectuée par les gestionnaires (acteur 1) du centre de météo, le service de gestion des ressources humaines (acteur 2) a décidé de modifier son interprétation (moment B) pour favoriser la livraison du service, à savoir de la production de données météo précises pour la défense nationale. Cette interprétation est venue d'une analyse de risque qui a démontré que bien qu'il était possible de se faire reprocher de ne pas être fidèle à la règle, la mise en œuvre de la politique publique constituait un poids suffisant pour ignorer une règle absurde vu le contexte. Cette situation a créé une grande frustration chez l'acteur 2 qui avait dû revoir son analyse et modifier son conseil.

Cette frustration provenait d'un paradoxe, à savoir que leur interprétation rigoriste ne semblait pas contribuer à la conformité que visaient précisément ces règles mêmes. Si l'acteur acceptait, vu son conditionnement culturel, de considérer des problèmes de cohérence dans la mise en œuvre des politiques publiques en calculant qu'il y avait tout de même plus davantage à faire ainsi pour protéger l'organisation, quel avantage y aurait-il à continuer d'agir ainsi si cette position légaliste ne protégeait justement plus l'organisation (Borry, 2015)?

\subsection{Deuxième cas : Une zone fermée}

\section{Contexte}

Une direction responsable des urgences environnementales désirait nommer un employé à partir d'un bassin de candidats qualifiés. Les postes affichés doivent indiquer où se situe le poste à doter, afin de clairement indiquer à d'éventuels postulants si un déménagement serait requis 
pour occuper le poste, advenant qu'ils aient été sélectionnés. Or, dans le cas qui nous intéresse, l'affiche indiquait que le poste doté serait à Ottawa et que d'autres postes pourraient être pourvus par le biais d'un bassin potentiel, sans préciser la localisation d'éventuelles nouvelles occasions d'emplois. À la suite de ce processus, un bassin de candidats pleinement qualifiés a été créé.

\section{Moment A}

Cette demande se frappa à un refus catégorique de la part des conseillers en ressources humaines (acteur 2) qui arguaient qu'une telle nomination serait faite contre les valeurs de la dotation dans la fonction publique, en particulier les valeurs d'accès et de transparence. Plus précisément, ils (acteur 2) avaient refusé au gestionnaire (acteur 1) l'utilisation de ce bassin puisque le poste avait été affiché pour Ottawa alors que la nouvelle opportunité était à Gatineau ${ }^{3}$. Pour ces conseillers (acteur 2), l'utilisation du bassin eût été contre l'esprit de la Loi sur l'emploi dans la fonction publique qui demande transparence et accès. Toujours selon leur raisonnement, il est possible que certains employés n'aient pas postulé pour un poste à Ottawa, mais qu'ils aient pu être intéressés à un poste à Gatineau. Ils (acteur 2) conseillèrent donc au gestionnaire (acteur 1), extrêmement frustré par le conseil reçu, d'afficher un nouveau processus, ce qui peut prendre aisément quatre mois. Ce retard de quatre mois aurait laissé un poste clé vacant. Incapable de recruter rapidement les employés nécessaires pour coordonner la réponse aux urgences environnementales au pays, cette division devenait à risque de ne pas pouvoir répondre rapidement et efficacement aux impératifs de la politique publique afférente.

\section{Réinterprétation (Moment B)}

À la suite des pressions du client (acteur 1) qui, pressé, insistait pour utiliser ledit bassin de candidats qualifiés, les conseillers en RH (Acteur 2) ont révisé leur interprétation pour permettre au gestionnaire de procéder à la nomination. Ce conseil était basé sur une nouvelle évaluation du risque. Cette nouvelle évaluation du risque, qui montrait, d'une part que la décision ne représentait aucun risque légal, tenait aussi compte du risque couru par le client, incapable de mettre en œuvre la politique publique pertinente.

\section{Méthodologie}

Notre étude de ces jeux d'acteurs se fond sur une démarche qualitative qui suit une logique inductive. La recherche qui soutient notre analyse s'appuie sur méthode d'analyse textuelle. Pour ce faire, nous avons analysé un corpus composé d'un total de 14 documents officiels. Ces documents ont été sélectionnés, à partir de tous les documents publics d'Environnement Canada, en vertu de leur pertinence pour observer des mécanismes en jeu chez les acteurs. Ils représentent l'ensemble de tous les écrits officiels nous permettant d'observer les interactions entre les gestionnaires-clients et les employés des ressources humaines, au cours de la période étudiée.

\footnotetext{
${ }^{3}$ Nota : La ville de Gatineau est reliée à la ville d'Ottawa par 4 ponts qui enjambe la rivière des Outaouais. Bien que constituant la frontière virtuelle entre le Québec et l'Ontario, les deux villes font partie de la même région administrative fédérale, à savoir la région de la Capitale nationale.
} 
Le corpus a été lu et analysé selon une méthode de codage ouvert (Lejeune, 2014), comprenant quatre étapes. La première étape, la micro-analyse consiste à lire attentivement et à annoter le corpus pour comprendre et synthétiser le contenu factuel.

La deuxième étape consiste à traiter le contenu en y apposant des étiquettes. Cette étape représente les briques de la construction de la recherche. L'étiquette, en définitive, porte sur le sens du communiqué et non sur les mots utilisés (Lejeune, 2014). Au terme de cet exercice, un total de quatorze étiquettes ont été identifiées.

Dans la troisième étape, nous avons procédé à la catégorisation des étiquettes, à savoir, le regroupement de celles-ci selon des catégories qui relient certains éléments, sans qu'ils présentent nécessairement les mêmes caractéristiques (Lejeune, 2014). De cet exercice, quatre catégories ont émergé.

La dernière étape fut la mise en rapport des étiquettes entre elles. $N^{\text {os }} 14$ étiquettes ont ainsi été placées dans un tableau en fonction de leur présence, ou de leur absence, dans chacun des 14 documents du corpus. Dans ce travail d'analyse, nous avons porté une attention particulière aux cas négatifs. En effet, comme le précise Lejeune (2014), l'analyse doit considérer l'analyse des cas opposés. Aussi, nous avons redivisé, lorsque les étiquettes le justifiaient, ces catégories en fonction de leur expression positive ou négative, subdivisant ainsi la catégorie en plusieurs sous-catégories, qui constituent nos étiquettes.

\section{5. Équilibre relationnel et contextuel : explorer l'antre de la Fabrique des politiques publiques.}

Le tableau qui suit montre le nombre d'occurrences par catégories obtenues par ce regroupement.

Tableau 1 : Étiquettes observées par catégorie

\begin{tabular}{|c|c|c|c|}
\hline Catégories & $\begin{array}{l}\text { Qualités } \\
\text { des étiquettes }\end{array}$ & $\begin{array}{l}\text { Nombre de documents } \\
\text { dans lesquels une } \\
\text { étiquette apparaît } \\
\text { au moins une fois } \\
\text { au moment } 1\end{array}$ & $\begin{array}{l}\text { Nombre de documents } \\
\text { dans lesquels une } \\
\text { étiquette apparaît } \\
\text { au moins une fois } \\
\text { au moment } 2\end{array}$ \\
\hline \multirow[t]{2}{*}{$\begin{array}{l}\text { Conformité } \\
\text { aux règles }\end{array}$} & $\begin{array}{l}\text { Étiquettes } \\
\text { positives }\end{array}$ & & \begin{tabular}{|l|l|l|}
1 & 1 & 1 \\
1 & 1 & 1 \\
1 & 1 & 1 \\
\end{tabular} \\
\hline & \begin{tabular}{|l|} 
Étiquettes \\
négatives
\end{tabular} & \begin{tabular}{|l|l|l|}
1 & 1 & 1 \\
1 & 1 & 1 \\
\end{tabular} & \\
\hline \multirow[t]{2}{*}{$\begin{array}{l}\text { Satisfaction } \\
\text { des clients }\end{array}$} & $\begin{array}{l}\text { Étiquettes } \\
\text { positives }\end{array}$ & & \\
\hline & $\begin{array}{l}\text { Étiquettes } \\
\text { négatives }\end{array}$ & \begin{tabular}{|l|l|l|}
1 & 1 & 1 \\
1 & 1 & 1 \\
\end{tabular} & \\
\hline $\begin{array}{l}\text { Satisfaction } \\
\text { du personnel RH }\end{array}$ & $\begin{array}{l}\text { Étiquettes } \\
\text { négatives }\end{array}$ & & |l \\
\hline Culture & $\begin{array}{l}\text { Étiquettes } \\
\text { négatives }\end{array}$ & 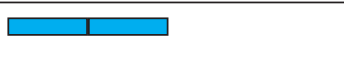 & 1 \\
\hline
\end{tabular}


On constate d'emblée une configuration différente au moment 1 et 2 . Les quatre catégories peuvent être regroupées en deux thèmes. Le premier concerne la satisfaction des acteurs eux-mêmes, c'est-à-dire les satisfactions des gestionnaires-clients (acteur 1) et du personnel des ressources humaines (acteur 2). Le second thème porte plutôt sur le contexte dans lequel les acteurs évoluent, c'est-à-dire la conformité aux règles et la culture.

Pour ce qui est des acteurs, on constate des résultats en porte-à-faux. Au moment 1 , l'insatisfaction marquée des acteurs 1 (quant au service reçu de l'acteur 2) est accompagnée d'une absence de signes de satisfaction ou d'insatisfaction chez l'acteur 2. Puis, au moment 2, la satisfaction relative de l'acteur 1 (en effet, on trouve des étiquettes positives et négatives) par rapport à ces mêmes services est accompagnée d'une insatisfaction chez l'acteur 2. Aussi, tout semble indiquer que la satisfaction des deux acteurs, pourtant appelés à collaborer, ne provient pas de la même source. Ce qui plaît à l'un semble déplaire, ou du moins laisser impassible, l'autre.

On trouve ainsi, d'une part, des gestionnaires qui cherchent à mettre en œuvre des politiques publiques tout en se soumettant à des impératifs de gestion (performance, pression, échéances serrées). D’autre part, les spécialistes en ressources humaines qui conseillent les gestionnaires quant à l'application des règles institutionnelles applicables à leur domaine. Cette dichotomie culturelle entraîne nécessairement une tension que nous avons en effet constatée dans nos résultats. Au moment 1, l'acteur 1 est insatisfait et l'acteur 2 est impassible, si l'on peut dire. Au moment 2, l'acteur 1 est plus satisfait et l'acteur 2 est insatisfait. La satisfaction des acteurs évolue de façon indépendante puisqu'elles répondent à des logiques différentes conditionnées par leurs référents culturels et leur réponse à l'environnement. Or, la satisfaction observée chez les gestionnaires (acteur 1) quant au service reçu des ressources humaines (acteur 2) est un indice du degré d'interférence ressenti par l'acteur 1 dans la mise en œuvre des politiques publiques.

Même si la nature de leur mandat leur impose des fins, et un contexte institutionnel différent, ils se doivent de collaborer. En effet, pour obtenir du succès, les gestionnaires (acteur 1) ont besoin de personnel compétent, au bon moment. Plus ils auront ce personnel rapidement, et en y consacrant le moins de temps possible, plus ils seront satisfaits. A contrario, plus ils rencontreront d'obstacles à recruter ou à promouvoir ce personnel, ou plus ils devront consacrer du temps à ces fonctions, moins ils seront efficaces dans la livraison de leur mandat ce qui influencera leur satisfaction. Or, en tant qu'acteurs de la mise en œuvre des politiques publiques, les gestionnaires sont soumis aux diverses règles institutionnelles qui entraînent des effets, néfastes ou non, sur leurs actions. Or ces règles sont interprétées par des professionnels (acteurs 2) qui conseillent les gestionnaires.

Lorsque deux acteurs devant collaborer regardent effectivement dans des directions opposées, la question de l'équilibre des forces se pose. Pour un ensemble de raisons, dont plusieurs qui sont liées à l'éthos de l'appareil public (Bozeman, 1993 ; Griggs et coll., 2015) et même à la nature des ressources humaines dans l'organisation (Le Gall, 2015; Dollinger, 2012), le conseil de l'acteur des ressources humaines prime habituellement sur celle du gestionnaire. Il est à noter que ce « conseil » des spécialistes des ressources humaines n'est pas une simple opinion, qui peut être balayée du revers de la main par le gestionnaire. La culture du gouvernement canadien élève à titre de quasi-institution les «conseils » des experts de ressources humaines qui bénéficient d'une très grande autorité morale. Un gestionnaire qui décide de ne pas suivre le « conseil » sera toujours à contre-courant de la 
culture ambiante. Bel exemple d'éléments culturels qui prend la forme et la force d'une véritable institution, il s'agit d'un construit propre à la fonction publique qui cherche à se protéger d'être pris en flagrant délit de ne pas avoir suivi les règles.

Pour résumer, on peut synthétiser la relation du moment 1 comme suit. Les gestionnaires dont la responsabilité est de mettre en œuvre des programmes, partie intégrante de politiques publiques, se trouvent obligés, en raison de la culture ambiante, de suivre des conseils péremptoires, qui peuvent parfois nuire à leur mandat, par des conseillers en ressources humaines, qui n'ont pas les mêmes objectifs. Cette situation de tension crée de l'insatisfaction chez l'acteur gestionnaire.

Pour ce qui est du second thème, à savoir l'aspect contextuel dans lequel les acteurs évoluent, la conformité aux règles est négative au moment 1 tout comme la culture. Cette combinaison de facteurs négatifs contribue à faire pression sur les acteurs qui y évoluent. Lorsque l'on met en parallèle les résultats des acteurs et ceux du contexte, on note certains regroupements utiles. Premièrement, la satisfaction, même mitigée, de l'acteur 1 est présente en même temps que la conformité aux règles. Deuxièmement, l'insatisfaction de l'acteur 2 cohabite avec la satisfaction de l'acteur 1 et la conformité aux règles. Notons aussi que la catégorie culture ne comporte que des étiquettes négatives et que celles - ci sont en plus grand nombre aux moments 2 , au moment où la satisfaction de l'acteur 1 , pourtant, apparaît. Au moment 2, la conformité devient positive alors que la culture continue à montrer des signes négatifs. Cela semble indiquer que la conformité n'est pas un facteur qui influence la culture. On le constate par différents éléments. Premièrement, la catégorie culture est négative dans les deux moments, peu importe la combinaison de configuration des autres catégories. Ni la satisfaction des acteurs ni la conformité aux règles ne semblent influencer positivement la culture. On est bien dans une tension culturelle.

La relation de satisfaction s'inverse chez les acteurs alors que la pression contextuelle se modifie (moment 2). Les étiquettes négatives font leur apparition dans la satisfaction des conseillers et des étiquettes positives apparaissent chez les gestionnaires. De plus, la catégorie contextuelle de conformité devient que positive, de toute négative qu'elle était. Quel est l'impact de ces éléments observés sur l'équilibre des forces dans la relation entre les acteurs?

Nous avons vu, avec Bourdieu (1980) et Potocki Malicet (1997), que les acteurs qui interprètent des règles chercheront à trouver des accommodements à celles-ci dans la mesure où ils croient qu'il est plus avantageux pour eux d'y désobéir que d'y obéir. Nous croyons que c'est bien cette dynamique qui a poussé les acteurs à interpréter les règles de manière plus souple au moment B.

Potocki Malicet (1997) avait établi que ce genre d'accommodements pouvait exister en vertu d'une certaine énergie propre à l'organisation, à savoir les objectifs de celle-ci (c.-à-d. les politiques publiques). Et cette attitude est facile à comprendre sous bien des aspects, car il s'agit du but même de l'organisation. Mais pourquoi à ce moment précis? Potocki Malicet (1997) a bien décrit les jeux d'équilibre à l'intérieur d'une organisation lorsque les règles internes en influencent négativement le fonctionnement :

"Toute organisation est un lieu d'équilibre entre les buts qui lui sont propres et les buts de ceux qui la constituent. Quand une règle ne permet pas d'effectuer les activités prescrites de façon adéquate, la pression qui naîtra de cette situation dysfonctionnelle n'aboutira pas à l'abandon de cette règle, mais elle fera l'objet d'aménagements de la part des acteurs. » (Potocki Malicet, 1997, p. 68) 
Cette notion de dysfonctionnement dépeint bien les tensions observées dans les relations entre les acteurs, c'est-à-dire la relation opposée entre la satisfaction des acteurs au moment 1 et au moment 2. Ces tensions habituelles étaient exacerbées par des pressions contextuelles militant en faveur de l'inversion de l'équilibre institutionnel. En effet, la pression provenant de l'insatisfaction des gestionnaires n'était pas isolée, mais agissait en parallèle avec les thèmes décrivant le contexte où s'agitent les acteurs. En effet, les deux catégories contextuelles, conformité aux règles et culture, ne présentent que des étiquettes négatives au moment 1 . Cette pression contextuelle se modifie puisqu'une des deux catégories, à savoir la conformité, devient positive.

Cet enjeu de conformité (voir moment 1) plaçait l'acteur des ressources humaines, dans une position très délicate et fort inconfortable face à l'acteur gestionnaire. Cette fragilité provenait d'un paradoxe, à savoir que leurs interprétations rigoristes ne semblaient pas (ou plus) contribuer à la conformité précisément visée par ces règles mêmes. Si l'acteur gestionnaire acceptait, vu son conditionnement culturel, de subir les conséquences des règles internes en raison de la nécessité de protéger l'organisation contre un manquement auxdites règles, quel avantage y avait-il à continuer d'agir ainsi si cette interprétation légaliste et pénalisante ne protégeait même plus l'organisation (Borry, 2015)? La valeur morale de l'acteur 2 s'amenuise devant cette double tension ce qui modifie l'équilibre des forces pour pousser l'acteur 1 à conclure que désobéir à la règle interne lorsque celle-ci était en interférence à la mise en œuvre des politiques publiques était plus profitable que de lui obéir.

\section{Conclusion}

Dans cet article, nous cherchions à comprendre l'impact des relations des fonctionnaires à titre d'acteurs dans la mise en œuvre des politiques publiques.

Dans un premier temps, par l'observation de cas réels, nous avons montré que les jeux d'acteurs pouvaient influencer significativement, voire mettre à risque, la mise en œuvre efficace des politiques publiques. Nous avons pu ainsi observer des acteurs internes (fonctionnaires) dont l'équilibre des relations peut influencer significativement la nature même des résultats obtenus. Les institutions internes conditionnent réellement l'agir des acteurs qui ne s'inscrivent pas toujours dans un mouvement linéaire qui serait en symbiose avec la finalité de l'action publique globale. Au contraire, leurs propres déterminants culturels déterminent une bonne partie de leurs actions. L'effet de leur rencontre, à l'intersection de mondes trop souvent parallèles, constitue un facteur non négligeable de la fabrique des politiques publiques.

Dans un second temps, une analyse documentaire nous a permis de replacer les relations entre acteurs dans un contexte institutionnel plus large. Une attention particulière à la satisfaction des différents acteurs a permis de faire ressortir des divergences majeures. À l'aide d'une lecture néo-institutionnaliste, nous avons établi que ces configurations de satisfaction répondaient à des cultures différentes, elles-mêmes animées par des mandats radicalement différents.

Leur collaboration mutuelle, forcée, est influencée par le contexte dans lequel ils évoluent et donc plus ou moins résistant aux règles internes. Si l'habitus culturel penche habituellement vers une interprétation stricto sensu des règles internes, l'insatisfaction des acteurs-gestionnaires, doublée de l'influence de pressions externes, peut inverser cet équilibre dans la relation des acteurs en créant des conditions propices à inciter les acteurs à trouver légitime de contourner la règle plutôt que de la suivre. 


\section{Bibliographie}

Arborio A. M., 2007. L'observation directe en sociologie : quelques réflexions méthodologiques à propos de travaux de recherches sur le terrain hospitalier. Recherche en soins infirmiers 90, 26-34.

Bernier L. et Lachapelle G., 1998. L'étude des politiques gouvernementales In Tremblay M., les politiques publiques canadiennes, Presses de l'Université Laval, Sainte-Foy,13-39.

Birkland T. A., 2005. Introduction to the Policy Process. M.E. Sharpe, Armonk.

Borry E. L., 2015. « Bureaucracy and Dissent» or « The Ethics of Red Tape »? Linking Red Tape and Guerrilla Government. Journal of Public Administration Research and Theory 25 (3), 996-1003.

Bourdieu P., 1980. Le sens pratique (le sens commun). Éditions de Minuit, Paris.

Bozeman B., 1993. A Theory of Government "Red Tape". Journal of Public Administration Research and Theory 3 (3), 273-304.

Bozeman B., 2000. Bureaucracy and Red Tape. Prentice Hall, Upper Saddle River.

De Ketele J. M. et Roegiers X., 2009. Méthodologie du recueil d'informations : Fondements des méthodes d'observation, de questionnaires, d'interviews et d'études de documents. De Boeck Supérieur, Paris.

De Ketele J.-M., 1980. Observer pour éduquer. Peter Lang, Berne.

Dollinger A., 2012. Pourquoi la loi sur la modernisation de la fonction publique a dérapé ? Optimum online 14 (1), 3- 3 .

Griggs V., Holden R., Rae J. and Lawless A., 2015. Professional Learning in Human Resource Management: Problematising the Teaching of Reflective Practice. Studies in Continuing Education 37 (2), 202-217.

Grossmann E., 2014. Acteur In Boussaguet L., Jacquot S. et Ravinet P., Dictionnaire Des Politiques Publiques. Presses De Sciences Po., Paris, 31- 38.

Howlett M., Ramesh M. and Perl A., 1995. Studying Public Policy: Policy Cycles \& Policy Subsystems. Oxford University Press, Toronto.

Katzenstein P. J., 1998. Cultural Norms and National Security: Police and Military in Postwar Japan. Cornell University Press, Ithaca.

Knoepfel P., Larrue C., Varone F. et Savard J. F., 2015. Analyse et pilotage des politiques publiques. Presses de l’Université du Québec, Québec.

Labolo M., 2013. Characteristic of Weber Bureucracy and Its Relevance in Indonesia. Asian Social Science 9 (2), 163-169.

Le Galès P., 2014. Gouvernance In Boussaguet L., Jacquot S. et Ravinet P., Dictionnaire des politiques publiques $3^{e}$ édition, Presses de Sciences Po., Paris, 299-308.

Le Gall J. M., 2015. Gestion des ressources humaines. Presses Universitaires de France. Paris.

Lecours A., 2002. Paradiplomacy: Reflections on the Foreign Policy and International Relations of Regions. International Negotiation 7 (1), 91-114.

Lejeune C., 2014. Manuel d'analyse qualitative: Analyser sans compter ni classer. De Boeck Supérieur, Louvain-La-Neuve.

Lemieux V., 2002. L'étude des politiques publiques (2e éd.). Presses de 1'Université Laval, Québec.

March J. G and Olsen J. P, 2006. Elaborating the «new Institutionalism » In Rhodes R. A. W., Binder S. A. and Rockman B. A., The Oxford Handbook of Political Institutions, Oxford University Press, Oxford, 3- 20.

Muller P. et Surel Y., 1998. L'analyse des politiques publiques. Montchrestien, Paris.

Pal L. A., 2006. Beyond Policy Analysis: Public Issue Management in Turbulent Times. Thompson \& Nelson, Toronto.

Pasmore W., 2006. Action Research in the workplace: the Socio-technical Perspective In Reason P. and Bradbury H., Handbook of Action Research: Concise Paperback Edition. SAGE, Newcastle upon Tyne. 
Pelletier R., 2005. Fédéralisme et fédérations : approches et pratiques In Imbeau L., Politiques publiques comparées dans les États fédérées : L'Allemagne, l'Australie, le Canada, les États-Unis et la Suisse, Presses de 1’Université Laval, Québec, 29-55.

Potocki Malicet D., 1997. Les règles de scolarité dans l'Université : importance et rôle des règles et des pratiques locales. Sociétés contemporaines 28 (1), 57-78.

Sabatier P. A., 1999. The need for better theories In Sabatier P. A., Theories of the Policy Process. Westview Press, Boulder, 3- 17. 
